Disease and 10\% had an alternative form of IBD (e.g. Proctitis, Lymphocytic Colitis or Collagenous Colitis). The ethnic mix in the responding cohort was 91\% Caucasian, $6 \%$ Asian, 2\% Mixed and 1\% was not stated. The sample had a mean score of $7.8(\mathrm{CI}=7-8.6)$. $98(40 \%)$ of patients' scores reflected "no depression"; 64 (26\%) reflected "mild depression"; 33 (14\%) reflected "moderate depression"; 36 (15\%) reflected "moderately severe depression"; 12 (5\%) of scores reflected "severe depression".

Conclusion $20 \%$ of our responding IBD patients were shown to have clinically significant levels of depression (moderately severe + severe), with $5 \%$ demonstrating scores suggestive of severe depression ( $1 \%$ expressing suicidal ideation). Relapse rates are known to be closely correlated with the severity of depression, and yet very few are on active treatment or review for this. The prevalence and severity of depression in our cohort of responding IBD patients supports the argument for screening all new IBD patients in order to optimise clinical well-being and treatment efficacy.

Disclosure of Interest None Declared.

\section{PTH-081 UNEARTHING THE TRUE PREVALENCE OF ANXIETY WITHIN A TYPICAL DISTRICT GENERAL COHORT OF INFLAMMATORY BOWEL DISEASE PATIENTS: IS IT TIME WE CONSIDERED ROUTINELY SCREENING FOR ANXIETY?}

doi:10.1136/gutjnl-2013-304907.568

1,"N Swart, 'D Wellsted, ${ }^{2} \mathrm{~K}$ Lithgo, ${ }^{2 \mathrm{~T}}$ Price, ${ }^{2} \mathrm{M}$ W Johnson. ${ }^{1}$ Centre for Lifespan and

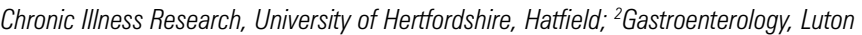
\& Dunstable University Hospital, Luton, UK

Introduction Previous studies have suggested that 15 to $30 \%$ of inflammatory bowel disease (IBD) patients also suffer from anxiety. Whilst most gastroenterologists would feel confident in recognising and diagnosing florid steroid induced psychosis, much of the associated anxiety experienced by IBD patients goes undiagnosed and untreated. Disease severity, recurrent flares, poor treatment adherence, disability, unemployment status, and socio-economic deprivation are all believed to be associated with anxiety in these patients. The severity of anxiety also appears to be directly correlated to the physical morbidity and malnutrition risk.

Objectives To assess the true prevalence of anxiety within our IBD patients.

Methods 2400 patients with IBD in the Luton \& Dunstable catchment were invited to participate in a web-based quality of life assessment, with the option to request a paper copy. Eligibility criteria required patients to be between 18 and 90 years of age, with no serious learning difficulties or pre-existing serious mental disorders. The well validated 7-item self-report "Generalised Anxiety Disorder (GAD) Questionnaire" was used. The GAD-7 has a minimum possible score of 0 and a maximum possible score of 21 . Scores of 5,10 , and 15 represent cut-off scores for mild, moderate, and severe anxiety.

Results 245 patients completed the assessment (43\% male; mean age $=53, \mathrm{SD}=17$ ). $45 \%$ had Ulcerative Colitis, $45 \%$ had Crohn's Disease and $10 \%$ had an alternative form of IBD (e.g. Proctitis, Lymphocytic Colitis, or Collagenous Colitis). The ethnic mix in the responding cohort was $91 \%$ Caucasian, $6 \%$ Asian, 2\% Mixed and 1\% was not stated. The sample had a mean score of $6.6(\mathrm{CI}=5.9-7.4)$. $72 \%$ of patients' scores reflected no anxiety or "mild anxiety"; $15 \%$ reflected "moderate anxiety"; and 13\% reflected "severe anxiety".

Conclusion $29 \%$ of our responding IBD patients were shown to have significant anxiety scores (moderate + severe), with 14\% demonstrating severe anxiety levels. Despite the severity, few of these patients were receiving treatment or therapy for their condition. The GAD score is a simple and quick tool that can be used in clinic. Given that anxiety is believed to directly affect the clinical course of $\mathrm{IBD}$, both the screening and treatment of this condition should be considered part of standard IBD medical care.

Disclosure of Interest None Declared.

\section{PTH-082 SERUM CALPROTECTIN: A NOVEL BIOMARKER TO PREDICT OUTCOME IN ACUTE SEVERE ULCERATIVE COLITIS?}

doi:10.1136/gutjnl-2013-304907.569

1." $\mathrm{N}$ C Hare, ' N A Kennedy, ${ }^{1} \mathrm{~K}$ Kingstone, 'I D Arnott, ' ${ }^{1} \mathrm{~A}$ G Shand, ${ }^{1} \mathrm{~K}$ R Palmer, ${ }^{1} \mathrm{D}$ Penman, ' $\mathrm{C}$ W Lees, '1 Satsangi. 'Gastrointestinal Unit, University of Edinburgh, Western General Hospital, Edinburgh, UK

Introduction Acute severe ulcerative colitis (ASUC) remains an important clinical problem and is associated with significant morbidity and requirement for colectomy. Faecal calprotectin and C-reactive protein (CRP) have previously been shown to predict the need for colectomy but there is an unmet need for further biomarkers. Serum calprotectin has not previously been analysed for this purpose and may provide a novel way of determining disease activity and outcome. The aim of this study was to assess how serum calprotectin relates to faecal calprotectin and other blood markers of inflammation, and to determine whether serum calprotectin on admission predicts colectomy.

Methods Blood samples were collected prospectively from patients who presented with ASUC as defined by the Truelove and Witts criteria. Blood samples were taken within the first 24 hours of admission. Faecal samples were pre-processed using $\mathrm{PhiCal}^{\mathrm{TM}}$ extraction buffer. Samples were stored at $-80^{\circ} \mathrm{C}$ and analysed in duplicate using the PhiCal ${ }^{\mathrm{TM}}$ calprotectin ELISA according to manufacturer's instructions. Samples with a calprotectin result of $>2500 \mu \mathrm{g} / \mathrm{g}$ were diluted and retested. Statistical comparisons were made between serum calprotectin and other markers of inflammation using Spearman's correlation coefficient, and ROC curve analysis was performed to determine how well each test performed in predicting colectomy.

Results There were 45 patients recruited to the study with ASUC, of which $22(49 \%)$ were female. Median age on admission was 40 years (interquartile range [IOR] 26-62). Median disease duration was 12 years (IOR 0-59). 26 of the 45 patients had a paired faecal sample for calprotectin analysis. There was no difference in sex, age or disease extent between those with or without faecal calprotectin. Serum calprotectin correlated significantly with CRP $\left(\mathrm{R}^{2}=0.46\right.$, $\mathrm{p}<0.0001)$ and with albumin $\left(\mathrm{R}^{2}=0.12, \mathrm{p}=0.023\right)$ but not with faecal calprotectin $\left(R^{2}=0.02, p=0.450\right)$. ROC analysis gave an AUC of 0.69 for serum calprotectin compared with 0.71 for CRP and 0.58 for faecal calprotectin. A cut off for serum calprotectin of $400 \mu \mathrm{g} / \mathrm{g}$ gave a sensitivity of 0.68 and a specificity of 0.69 .

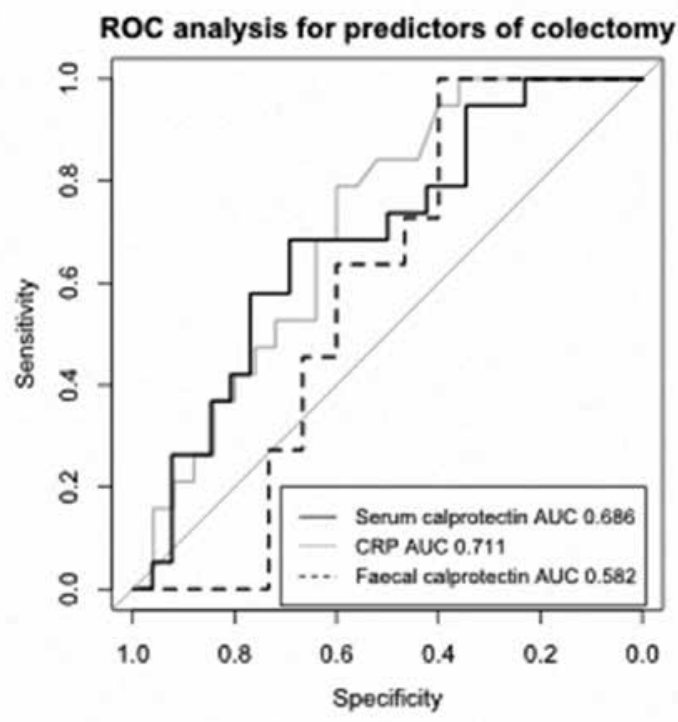

Abstract PTH-082 Figure 1 